\title{
AN EXPERIMENTAL ANALYSIS OF THE POWER CONSUMPTION OF CONVOLUTIONAL NEURAL NETWORKS FOR KEYWORD SPOTTING
}

\author{
Raphael Tang Weijie Wang Zhucheng Tu Jimmy Lin \\ David R. Cheriton School of Computer Science \\ University of Waterloo \\ \{r33tang, w268wang, michael.tu, jimmylin\}@uwaterloo.ca
}

\begin{abstract}
Nearly all previous work on small-footprint keyword spotting with neural networks quantify model footprint in terms of the number of parameters and multiply operations for a feedforward inference pass. These values are, however, proxy measures since empirical performance in actual deployments is determined by many factors. In this paper, we study the power consumption of a family of convolutional neural networks for keyword spotting on a Raspberry Pi. We find that both proxies are good predictors of energy usage, although the number of multiplies is more predictive than the number of model parameters. We also confirm that models with the highest accuracies are, unsurprisingly, the most power hungry.
\end{abstract}

Index Terms - keyword spotting, power consumption

\section{INTRODUCTION}

Conversational agents that offer speech-based interfaces are increasingly part of our daily lives, both embodied in mobile phones as well as standalone consumer devices for the home. Prominent examples include Google's Assistant, Apple's Siri, Amazon's Alexa, and Microsoft's Cortana. Due to model complexity and computational requirements, full speech recognition is typically performed in the cloud: recorded audio is transferred to a datacenter for processing. For both practical and privacy concerns, devices usually perform keyword spotting locally to detect a trigger phrase such as "hey Siri", which provides an explicit acknowledgment that subsequent audio recordings of user utterances will be sent to backend servers and thus may be logged and analyzed. Beyond detecting these triggers, it makes sense to perform recognition of simple commands such as "go" and "stop" as well as common responses such as "yes" and "no" directly on-device. Together, these represent instances of the keyword spotting task on continuous speech input. Due to power constraints on mobile devices, it is desirable that such keyword spotting models are "compact" and have a "small footprint" (which we formally define below).

Over the past several years, neural networks have been successfully applied to the keyword spotting task (see more details in Section 27. When discussing the "footprint" of a model, the literature usually refers to two easily quantifiable values: the number of model parameters and the number of multiplies for a feedforward inference pass. Model "compactness" is thus measured in terms of these two quantities, which are of course proxies at best. Ultimately, what matters most is the energy consumption during inference.

To our knowledge, previous work in keyword spotting stops short of actual energy measurements. Thus, the primary contribution of this paper is the deployment of a number of convolutional neural networks for keyword spotting on a Raspberry Pi, where we are able to measure the energy usage of various models and relate these measurements back to the proxies used in previous work. We find that the number of multiplies does indeed predict energy usage and model latency, as does the number of parameters (albeit the relationship is weaker). Therefore, in the absence of actual power measurements, these proxies can be helpful in guiding model development, although we advise caution in interpreting both measures. Finally, as expected, we confirm that the most accurate models are also the most power hungry, suggesting unavoidable tradeoffs with this family of $\mathrm{CNN}$ architectures.

\section{RELATED WORK}

The application of neural networks to keyword spotting, of course, is not new. Chen et al. [1] introduced multi-layer perceptrons as an alternative to HMM-based approaches. Sainath and Parada [2] built on that work and achieved better results using convolutional neural networks (CNNs). They specifically cited reduced model footprints (for low-power applications) as a major motivation in moving to CNNs.

Despite more recent work in applying recurrent neural networks to the keyword spotting task [3, 4], we focus on the family of CNN models for several reasons. CNNs today remain the standard baseline for small-footprint keyword spotting-they have a straightforward architecture, are relatively easy to tune, and have implementations in multiple deep learning frameworks.

In this paper, we do not propose any new models for 


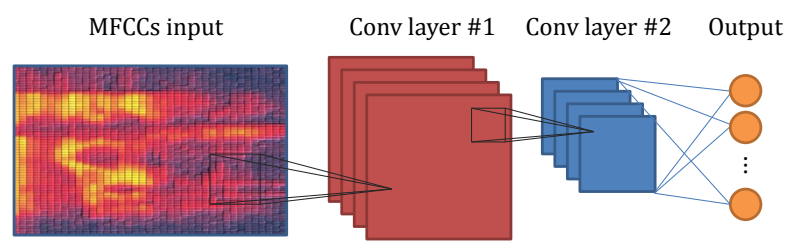

Fig. 1: Convolutional neural network architecture for keyword spotting.

\begin{tabular}{r|ccccc|cc}
\hline type & $m$ & $r$ & $n$ & $p$ & $q$ & Par. & Mult. \\
\hline conv & 20 & 8 & 64 & 1 & 3 & $10.2 \mathrm{~K}$ & $27.7 \mathrm{M}$ \\
conv & 10 & 4 & 64 & 1 & 1 & $164 \mathrm{~K}$ & $95.7 \mathrm{M}$ \\
lin & - & - & 32 & - & - & $1.20 \mathrm{M}$ & $1.20 \mathrm{M}$ \\
dnn & - & - & 128 & - & - & $4.1 \mathrm{~K}$ & $4.1 \mathrm{~K}$ \\
softmax & - & - & $n_{\text {labels }}$ & - & - & $1.54 \mathrm{~K}$ & $1.54 \mathrm{~K}$ \\
\hline \hline Total & - & - & - & - & - & $1.37 \mathrm{M}$ & $125 \mathrm{M}$ \\
\hline
\end{tabular}

Table 1: Structure of the cnn-trad-fpool3 model.

keyword spotting. Instead, we conducted a thorough experimental analysis of the power consumption of CNNs proposed by Sainath and Parada [2], using Google's recently-released Speech Commands Dataset [5] as the benchmark.

Canziani et al. [6] previously studied the relationship between power consumption, inference time, and accuracy using deep neural networks for computer vision tasks running on an NVIDIA Jetson TX1 board. This work studies many of these same relationships for keyword spotting, but on a more wimpy device, the Raspberry Pi.

\section{EXPERIMENTAL DESIGN}

All experiments described in this paper were conducted with Honk, our open-source PyTorch reimplementation of public TensorFlow keyword spotting models, which are in turn based on the work of Sainath and Parada [2]. We have confirmed that our PyTorch implementation achieves the same accuracy as the original TensorFlow references [7]. All our code is available on GitHub ${ }^{1}$ for others to build upon.

\subsection{Model Description}

For feature extraction, we first apply a band-pass filter of $20 \mathrm{~Hz} / 4 \mathrm{kHz}$ to the input audio to reduce noise. Fortydimensional Mel-Frequency Cepstrum Coefficient (MFCC) frames are then constructed and stacked using a $30 \mathrm{~ms}$ window and a $10 \mathrm{~ms}$ frame shift. All frames are stacked across a $1 \mathrm{~s}$ interval to form the two-dimensional input to our models.

The basic model architecture for keyword spotting, shown in Figure 1. comprises one or more convolutional layers followed by fully-connected hidden layers, ending with a soft-

\footnotetext{
${ }^{1}$ https://github.com/castorini/honk
}

\begin{tabular}{r|ccccc|cc}
\hline type & $m$ & $r$ & $n$ & $p$ & $q$ & Par. & Mult. \\
\hline conv & 21 & 8 & 94 & 2 & 3 & $15.8 \mathrm{~K}$ & $42.2 \mathrm{M}$ \\
conv & 6 & 4 & 94 & 1 & 1 & $212 \mathrm{~K}$ & $60.2 \mathrm{M}$ \\
lin & - & - & 32 & - & - & $854 \mathrm{~K}$ & $854 \mathrm{~K}$ \\
dnn & - & - & 128 & - & - & $4.1 \mathrm{~K}$ & $4.1 \mathrm{~K}$ \\
softmax & - & - & $n_{\text {labels }}$ & - & - & $1.54 \mathrm{~K}$ & $1.54 \mathrm{~K}$ \\
\hline \hline Total & - & - & - & - & - & $1.09 \mathrm{M}$ & $103 \mathrm{M}$ \\
\hline
\end{tabular}

Table 2: Structure of the cnn-tpool2 model.

\begin{tabular}{r|ccccccc|cc}
\hline type & $m$ & $r$ & $n$ & $p$ & $q$ & $s$ & $v$ & Par. & Mult. \\
\hline conv & 101 & 8 & 186 & 1 & 1 & 1 & 1 & $150 \mathrm{~K}$ & $4.99 \mathrm{M}$ \\
dnn & - & - & 128 & - & - & - & - & $786 \mathrm{~K}$ & $786 \mathrm{~K}$ \\
dnn & - & - & 128 & - & - & - & - & $16.4 \mathrm{~K}$ & $16.4 \mathrm{~K}$ \\
softmax & - & - & $n_{\text {labels }}$ & - & - & - & - & $1.54 \mathrm{~K}$ & $1.54 \mathrm{~K}$ \\
\hline \hline Total & - & - & - & - & - & - & - & $954 \mathrm{~K}$ & $5.76 \mathrm{M}$ \\
\hline
\end{tabular}

Table 3: Structure of the cnn-one-stride1 model.

max output. More specifically, an input of MFCCs $\mathbf{X} \in \mathbb{R}^{t \times f}$ is convolved with weights from the first convolutional layer, $\mathbf{W} \in \mathbb{R}^{m \times r \times n}$, where $t$ and $f$ are the lengths in time and frequency, $m$ and $r$ are the width and height of the convolution filter, and $n$ is the number of feature maps. If desired, the convolution can stride by $s \times v$ and max-pool in $p \times q$, parameters which also affect the compactness of the model. Rectified linear units are used as the activation function for each non-linear layer.

From this basic design, Sainath and Parada [2] proposed a number of specific models. We evaluated the following:

- trad-fpool3: The base model, illustrated in Table 1 comprises two convolution layers followed by a linear layer, a hidden layer, and a final softmax layer. All other variants are derived from this model.

- one-fstride $\{4,8\}$ : Limiting the number of multiplies and parameters, these are compact variants that stride in frequency and also use only one convolution layer. Sainath and Parada found that one-fstride 4 performs better than one-fstride8.

- tpool $\{2,3\}$ : These are variants that pool in time. Sainath and Parada found that, depending on the task, tpool2 has performance equivalent to or better than trad-fpool3. See Table 2 for the parameter breakdown of tpool2.

- trad-poo12: TensorFlow's variant of the base model trad-fpool3, with comparable accuracy, but using fewer multiplies.

- one-stride1: TensorFlow's compact variant of one-fstride 4 (detailed in Table 3). It uses a standard striding of $1 \times 1$ and thus has more parameters and multiplies, but achieves better accuracy. 


\begin{tabular}{lr|rr|rrr}
\hline Model & Test Accuracy & Par. & Mult. & Latency/q (ms) & Energy/q (mJ) & Peak Power (W) \\
\hline one-fstride4 & $70.28 \%$ & $220 \mathrm{~K}$ & $1.43 \mathrm{M}$ & 40 & 28 & 0.99 \\
one-fstride8 & $67.90 \%$ & $337 \mathrm{~K}$ & $1.43 \mathrm{M}$ & 42 & 29 & 1.02 \\
\hline one-stride1 & $77.06 \%$ & $954 \mathrm{~K}$ & $5.76 \mathrm{M}$ & 100 & 115 & 1.52 \\
trad-pool2 & $87.51 \%$ & $1.38 \mathrm{M}$ & $98.8 \mathrm{M}$ & 146 & 306 & 2.60 \\
\hline tpool2 & $91.97 \%$ & $1.09 \mathrm{M}$ & $103 \mathrm{M}$ & 204 & 384 & 2.21 \\
tpool3 & $91.23 \%$ & $823 \mathrm{~K}$ & $73.7 \mathrm{M}$ & 159 & 279 & 2.16 \\
\hline trad-fpool3 & $89.43 \%$ & $1.37 \mathrm{M}$ & $125 \mathrm{M}$ & 227 & 431 & 2.20 \\
\hline Feature extraction only & - & - & - & 31 & 19 & 0.80 \\
\hline
\end{tabular}

Table 4: Performance of CNN variants on the Raspberry Pi in terms of accuracy, footprint, latency, and power consumption. The compact model is one-stridel and the full model is trad-pool2. For reference, we also include a condition that only performs feature extraction. Energy calculations and peak power exclude idle power draw of $1.9 \mathrm{~W}$.

\subsection{Model Export and Inference}

To run model inference on the Raspberry $\mathrm{Pi}$, we exported Honk models written and trained in PyTorch to Caffe2 using ONNX ${ }^{2}$ the Open Neural Network Exchange format used for interchanging models between different deep learning frameworks. While PyTorch is good for research and rapidly iterating on model architecture, it was not designed to serve models in deployment settings, unlike Caffe2, which supports running deep learning models on production servers as well as mobile devices and Raspbian. This feature makes Caffe2 especially useful for evaluating keyword spotting models in environments where they will actually be deployed. The practice of building and training models in one framework and running inference in another has been used in production at Facebook. We built Caffe2 from source for Raspbian, with the $-\mathrm{mf} p u=$ neon flag, to specify the use of NEON (ARM's Advanced SIMD) optimizations. Our models use 32-bit floating point operations and $\mathrm{Caffe} 2$ implements convolutions using the im $2 \mathrm{col}$ approach.

Evaluation was performed on a Raspberry Pi 3 Model B (ARM Cortex-A53) running Raspbian Stretch (4.9.41-v7+). On the Raspberry Pi, we run a Caffe2 service which imports an ONNX model and performs inference (as described above). To capture power measurements, the Raspberry Pi is plugged into a Watts Up Pro meter, which has a USB port from which measurements can be programmatically read. Power measurements are taken at a frequency of $1 \mathrm{~Hz}$ from an external laptop connected to the meter. The length of each experimental trial is sufficiently long (on the order of minutes) that this resolution yields reasonably accurate measurements. During each experimental trial, a script on the Raspberry Pi iterates through all keyword classes for a fixed model, calling an API served by the laptop to start and stop measurements before and after the Caffe2 service call. Each Caffe2 service call evaluates all test examples for a given keyword class. There were a total of 2,567 test examples for all keyword classes combined.

\footnotetext{
${ }^{2}$ http://onnx.ai/
}

\section{EXPERIMENTAL RESULTS}

We evaluated the convolutional neural networks described in the previous section using Google's Speech Commands Dataset [5], which was released in August 2017 under a Creative Commons license 3 The dataset contains 65,000 one-second long utterances of 30 short words by thousands of different people, as well as such background noise samples as pink noise, white noise, and human-made sounds.

The Google blog post also references the TensorFlow implementation of Sainath and Parada's models, which we have ported to PyTorch and validated their correctness [7]. For consistency, we evaluated our PyTorch implementations, but otherwise followed exactly the same experimental setup as Google's reference. Specifically, our task is to classify a short one-second utterance as "yes", "no", "up", "down", "left", "right", "on", "off", "stop", "go", silence, or unknown. As the focus of this paper is on model performance in terms of energy usage and not on accuracy per se, we refer interested readers to details in Tang and Lin [7]. Our evaluation metric is accuracy, which is simply measured as the fraction of classification decisions that are correct.

Our main results are shown in Table 4 For each model, we show its accuracy on the test set and its model footprint in terms of the number of model parameters and the number of multiplies required for a feedforward inference pass. The next columns show the average query latency for each model on a test instance, the energy per query, and the peak power draw during the experimental run. The energy calculations as well as the peak power figures exclude energy consumed by the Raspberry Pi in its idle state, which has a power draw of $1.9 \mathrm{~W}$. For reference, we also report a condition that performs only feature extraction.

We found strong evidence of a positive linear relationship between the number of multiply operations used in the models and the energy used per query $\left(R^{2}=0.9641, p=0.0001\right)$ in Figure 2 (left) and also between the number of multiplies and latency per query $\left(R^{2}=0.8863, p=0.0015\right)$ in Figure 2 (right). There is also strong evidence of a positive relation-

\footnotetext{
${ }^{3}$ https://research.googleblog.com/2017/08/
} 

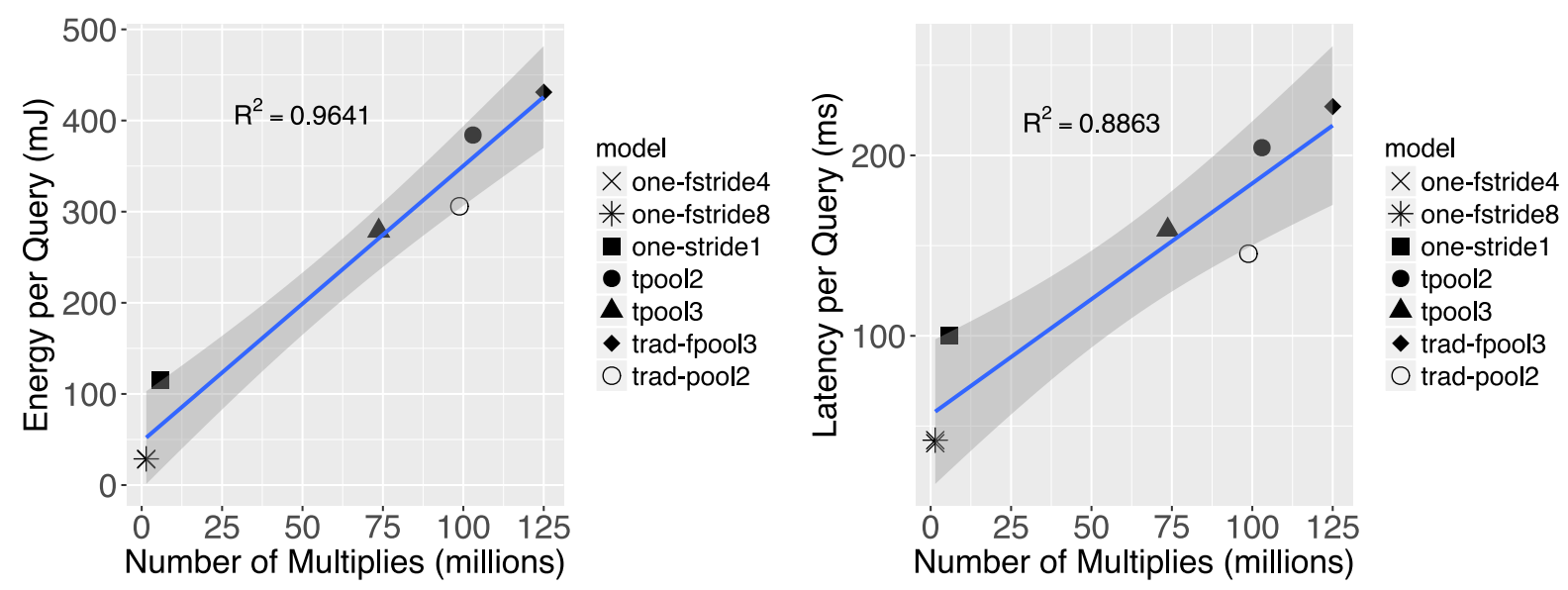

Fig. 2: Energy (left) and latency (right) per query vs. number of multiplies, with the $95 \%$ confidence interval.

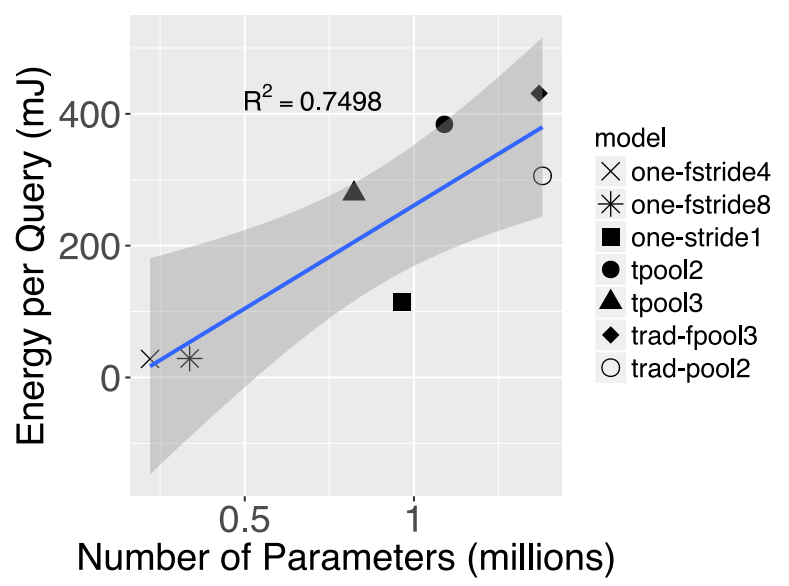

Fig. 3: Energy per query vs. number of parameters (95\% CI).

ship between the number of parameters and the energy used per query $\left(R^{2}=0.7498, p=0.0118\right)$ in Figure 3 and between the number of parameters and latency per query $\left(R^{2}=\right.$ 0.7237, $p=0.0152$ ), not shown. However, the strength of correlations for the number of parameters is weaker.

These results suggest that the number of multiplies, and to a lesser extent, the number of parameters, are useful proxy measures when developing small-footprint keyword spotting models that optimize for power consumption. Nevertheless, we suggest that these metrics must still be interpreted with caution. For example, we see that two models with similar numbers of multiplies can still have very different energy profiles: tpool2 and trad-pool2 have comparable numbers of multiplies but the former is $40 \%$ slower and consumes $25 \%$ more energy per query. However, the latter has a higher peak power draw.

Finally, we plot the relationship between energy usage and model accuracy in Figure 4 The strong correlation observed $\left(R^{2}=0.8919, p=0.0014\right)$ suggests that "you get

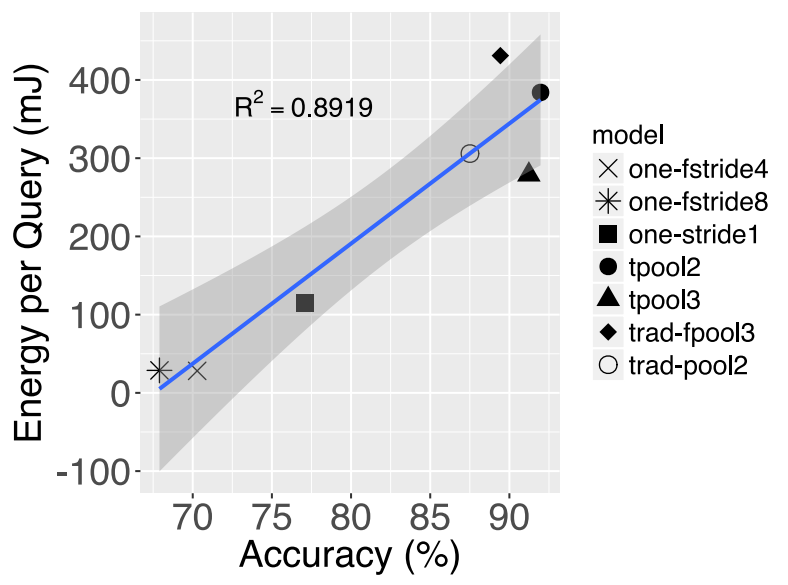

Fig. 4: Energy per query vs. accuracy (95\% CI).

what you pay for", in the sense that at least for this family of models, a designer must trade off accuracy for power consumption, and the relationship is surprisingly linear.

\section{CONCLUSIONS}

In this paper, we close a gap in the literature on smallfootprint keyword spotting. Previous work adopts the number of model parameters and multiplies in an inference pass as optimization objectives, under the assumption that smaller values translate into lower power consumption. To our knowledge, this assumption has not actually been verified until now. We do indeed find that both metrics are strong predictors of energy usage, although the number of multiplies exhibits a stronger correlation. While both are useful proxies during model development, we noticed sufficient variations-for example, models with similar multiplies but very different performance profiles-that actual power measurements may still be required for conclusive summative evaluations. 


\section{REFERENCES}

[1] Guoguo Chen, Carolina Parada, and Georg Heigold, "Small-footprint keyword spotting using deep neural networks," in ICASSP, 2014, pp. 4087-4091.

[2] Tara N. Sainath and Carolina Parada, "Convolutional neural networks for small-footprint keyword spotting," in Interspeech, 2015, pp. 1478-1482.

[3] Sercan Ömer Arik, Markus Kliegl, Rewon Child, Joel Hestness, Andrew Gibiansky, Christopher Fougner, Ryan Prenger, and Adam Coates, "Convolutional recurrent neural networks for small-footprint keyword spotting," arXiv:1703.05390 3, 2017.

[4] Ming Sun, Anirudh Raju, George Tucker, Sankaran Panchapagesan, Gengshen Fu, Arindam Mandal, Spyros Matsoukas, Nikko Strom, and Shiv Vitaladevuni, "Max-pooling loss training of Long Short-Term Memory networks for small-footprint keyword spotting," arXiv:1705.02411 1, 2017.

[5] Pete Warden, "Launching the speech commands dataset," Google Research Blog, 2017.

[6] Alfredo Canziani, Adam Paszke, and Eugenio Culurciello "An analysis of deep neural network models for practical applications," arXiv:1605.07678, 2016.

[7] Raphael Tang and Jimmy Lin, "Honk: A PyTorch reimplementation of convolutional neural networks for keyword spotting," arXiv:1710.06554v2, 2017. 